\title{
Isolation and characterisation of glyphosate-degrading bacteria isolated from local soils in Malaysia
}

\begin{abstract}
Glyphosate ( $N$-(phosphonomethyl)glycine) is a herbicide that is used to kill broadleaf weeds and grass, which was developed by the Monsanto company in the early 1970s. However, excessive usage of glyphosate in the agricultural field has led to soil accumulation of the pesticides causing various adverse effects. Due to the concern on its toxicity, an alternative way to ease its effect is through bioremediation, which exploits the ability of microorganisms to degrade harmful toxic substances into less toxic forms. Seven different microbial strains were isolated from glyphosate-contaminated sites in Malaysia. These strains were able to grow in a medium containing glyphosate as the sole phosphorus source. None of the strains were able to grow when glyphosate was introduced as a sole carbon source. Two microbial strains: AQ5-12 and AQ5-13, were found to be the best degraders among them as they were able to withstand up to $12 \mathrm{ml} / \mathrm{L}$ of the Roundup ${ }^{\circledR}$ glyphosate formulation and $200 \mathrm{ppm}$ of analytical grade glyphosate. Based on 16s rRNA and biochemical tests, AQ5-12 was identified as Burkholderia vietnamiensis, whereas AQ5-13 was identified as Burkholderia sp. AQ5-12, with the latter possessing better degradation capability compared to AQ5-13 with utilisation of 91 and $74 \%$ of $50 \mathrm{ppm}$ glyphosate, respectively. The best optimum degradation conditions were seen at the temperature of $30{ }^{\circ} \mathrm{C}$ and a $\mathrm{pH}$ of 6 for both strains. Based on these results, both strains displayed their potential to be used in the bioremediation of glyphosate-contaminated environment.
\end{abstract}

Keyword: Glyphosate; Biodegradation; Burkholderia vietnamiensis; Burkholderia sp.; Cultivation conditions 